\title{
A GESTÃO DO CONHECIMENTO PESSOAL E SEU PAPEL NO APRENDIZADO CONTÍNUO E SUCESSO NA ERA DAS ACELERAÇÕES
}

\author{
PERSONAL KNOWLEDGE MANAGEMENT AND ITS ROLE IN CONTINUOUS LEARNING AND \\ SUCCESS IN THE ERA OF ACCELERATION
}

Victor Couto Alves ${ }^{1}$

Luciana Martins Oliveira da Silva²

\section{RESUMO}

O mundo em constante aceleração tem gerado impactos tanto sociais como organizacionais. No centro disso tudo está o indivíduo e sua necessidade de estar continuamente desenvolvendo-se, afinal, é ele o componente principal das sociedades e das organizações. Porém, para que o indivíduo consiga desenvolver essa estabilidade dinâmica, ou seja, a capacidade de estar continuamente desenvolvendose e adaptando-se a esse mundo, é necessário repensarmos como ele aprende e utiliza metodologias que o auxiliem no controle do seu desenvolvimento. Nesse sentido, objetivou-se estudar o papel da gestão do conhecimento pessoal nesse processo, por meio da revisão bibliográfica em livros, artigos, sites e revistas sobre o assunto. Como resultado, verificou-se de fato como a gestão do conhecimento pessoal contribui para a clareza e responsabilidade do indivíduo, servindo como um mapa de orientação, e não necessariamente como a solução do problema, cabendo ao indivíduo agir para atingir o objetivo desejado.

Palavras-chave: Gestão do Conhecimento Pessoal, Conectivismo, Era das Acelerações, Aprendizagem, Conhecimento

\section{ABSTRACT}

The world in constant acceleration has generated both social and organizational impacts. At the center of all this is the individual and his need to be continually developing, after all, he is the core of societies and organizations. However, in order for the individual to be able to develop this dynamic stability, that is, the capacity to be continuously developing and adapting to this world, it is necessary to rethink how he learns and uses methodologies that help him to control his development. In this sense, the objective was to study the role of personal knowledge management in this process, through a bibliographic review in books, articles, websites and magazines on the subject, As a result, it has actually been verified how the management of personal knowledge contributes to the clarity and responsibility of the individual, serving as a guiding map, not necessarily as the solution of the problem, and it is up to the individual to act to achieve the desired goal.

Keywords: Personal Knowledge Management, Connectivism, Age of Accelerations, Learning, Knowledge

\footnotetext{
${ }^{1}$ Pós-graduação em Gestão do Conhecimento e Inteligência Empresarial (UFRJ) - Rio de Janeiro, Brasil. E-mail: vcoutoalves@gmail.com

2 Pós-graduação em Gestão do Conhecimento e Inteligência Empresarial (UFRJ) - Rio de Janeiro, Brasil. E-mail: martins@peq.coppe.ufrj.br
} 


\section{INTRODUÇÃO}

\subsection{Contextualização do Tema e Apresentação do Problema}

A mudança que nossa sociedade está passando talvez seja como quando fizemos a transição entre a economia agrícola para a revolução industrial (PALMER e BLAKE, 2018). Compara-se também à quando mudamos de uma sociedade predominantemente oral e tribal para uma sociedade escrita e institucional, e quando a imprensa de Gutenberg nos transformou em uma sociedade com a mídia impressa e de massa. Agora a mídia elétrica e eletrônica está nos transferindo para uma sociedade digital e em rede. (JARCHE, 2014). Isso deve-se principalmente pois "a armazenagem e a disseminação de informações se tornaram praticamente gratuitos", afetando diretamente as relações humanas (FRIEDMAN, p. 1168).

Levy (2010) nos alerta que diante deste "imenso mar de informações" ficamos sem saber ao certo o que fazer. No passado, antes do meio digital, isso não acontecia porque a criação e o gerenciamento do fluxo dessa informação eram feitos pelas igrejas, as escolas e até mesmo a mídia, ou seja, eles que de certa forma eram detentores e criadores da informação e consequentemente, diziam o que era ou não verdade, e no que deveríamos acreditar ou não. Desta forma, cabia a nós apenas construir nosso conhecimento a partir do que nos era enviado. Atualmente, no entanto, com o número de conexões que fazemos e com o acesso à informação muito mais fácil, temos, ou pelo menos deveríamos ter, a responsabilidade de gerenciar esse fluxo da informação.

Para Levy (2010) isso é um "problema da gestão do conhecimento pessoal", pois praticando-a seremos capazes de aprender a ter uma atitude mais responsável diante desse cenário. Nesta mesma linha, Jarche (2019), complementa que a Gestão do Conhecimento Pessoal é uma maneira de navegar nesse grande mar de informação e conhecimento pois através dela podemos desenvolver práticas individuais para um melhor sensemaking, unir comunidades de prática para nos guiar e interagir com redes sociais profissionais para nos manter relevantes.

\subsection{Problema}

O artigo tem como tema a gestão do conhecimento pessoal e seu papel no aprendizado contínuo e sucesso na sociedade atual onde o fluxo de informação está cada vez maior e mais rápido. Assim, o problema consiste na seguinte questão: Como a prática da gestão do conhecimento pessoal pode contribuir para o desenvolvimento pessoal, profissional, e para a resiliência na era digital? 


\subsection{Objetivos}

\subsubsection{Objetivo Principal}

O artigo tem por objetivo identificar as principais características da gestão do conhecimento pessoal e como ela pode contribuir para que as pessoas consigam gerenciar de forma efetiva a si mesmas assim como os fluxos de informações aos quais são submetidas.

\subsubsection{Objetivos Secundários}

a) Discorrer sobre o contexto atual que vivemos: a era das acelerações e da abundância e seus desafios.

b) Mostrar como esse contexto atual está impactando na forma como educamos e aprendemos.

c) Contextualizar a gestão do conhecimento e sua importância na aprendizagem.

d) Apresentar o que é a gestão do conhecimento pessoal e seus principais modelos.

\subsection{Justificativa}

A necessidade de se discutir a Gestão do Conhecimento Pessoal não é recente. Segundo Swigon (2010), a história da mesma começa com as ideias do Drucker, ainda em 1968, sobre o desafio de gerenciar a produtividade dos trabalhadores do conhecimento como parte central da gestão nas organizações.

Frand e Hixon, por exemplo, introduziram o conceito para "ajudar estudantes a adquirir competências informacionais necessárias para serem bem-sucedidos no cenário de negócios global que emergira, de forma a lidar melhor com a informação e seu excesso e transformar pedaços de informação em algo mais sistemático, de forma a expandir o conhecimento pessoal" (SWIGON, 2010).

Ainda no âmbito acadêmico, Dorsey e seus colegas também descreveram a Gestão do Conhecimento Pessoal como um framework para ajudar estudantes de graduação em 3 diferentes áreas: 1) para melhorar o senso de responsabilidade como estudante; 2) como um auxílio para integrar o currículo acadêmico (disciplinas gerais e específicas); 3) como uma abordagem para ajudar na integração de iniciativas de tecnologia ao longo do currículo estudantil. (SWIGON, 2010).

No entanto, não é só entre profissionais e estudantes que o tema se faz relevante, sendo importantíssimo para a adaptação em face das mudanças que o mundo enfrenta. (SWIGON, 2013).

Com o auxílio da tecnologia, por exemplo, a gestão do conhecimento pessoal ainda pode ser uma assistente para nosso cérebro. Tiago Fortes (2019) por exemplo desenvolveu uma 
metodologia chamada "Building A Second Brain" no qual utiliza técnicas de gestão do conhecimento pessoal e ferramentas digitais de captura, organização e compartilhamento de informação, que é capaz de melhorar consideravelmente a gestão do fluxo da informação. Segundo ele, isso acontece, pois, nosso cérebro tem uma limitação: "o cérebro é para ter ideias e não para armazená-los", por isso, a necessidade de utilizar um "segundo cérebro", que neste caso são as ferramentas.

Por fim, a Gestão do Conhecimento Pessoal "pode ser considerada uma forma de gestão de carreira e vida pessoal" (GORMAN e PAULEEN, 2011 apud SOUSA, RAMOS FILHO E VASCONCELOS, 2014, s.p.).

\subsection{Estrutura do Trabalho}

O artigo é apresentado em três partes. A parte I é composta pela introdução, que contém a contextualização e apresentação do tema, o objetivo final, objetivos intermediários e a justificativa do trabalho.

Na parte II, são apresentados os referenciais teóricos, através de uma revisão de literatura abrangendo assuntos que ajudam a entender o tema. Primeiramente é contextualizado o mundo que vivemos hoje, apresentando os diferentes conceitos que são utilizados para referencia-lo, com destaque para a Era das Acelerações e Era da Abundância, e seus respectivos desafios em geral. Em seguida, foca-se nas transformações da economia e na educação causadas por esse novo mundo. Como resposta à essas transformações, apresenta-se os conceitos, modelos e a importância da gestão do conhecimento e da gestão do conhecimento pessoal, com foco na metodologia Seek, Sense e Share do Harold Jarche.

Na parte III, são feitas as considerações finais e sugestões adicionais de trabalho. 


\section{REFERENCIAL TEÓRICO}

\subsection{Era das Acelerações e da Abundância: $O$ que é e quais são os Desafios.}

Muitos referem-se a esse novo mundo de diferentes formas, mas com significados similares ou complementares. Mundo VUCA, por exemplo, foi introduzida pelo U.S. Army War College para descrever o mundo multilateral mais volátil, incerto, complexo e ambíguo que resultou do fim da Guerra Fria. Diamandis e Kotler (2018) chamam de Era da Abundância, ou seja, um mundo de muitas possibilidades, no qual pela primeira vez na história poderemos alcançar novos patamares, por meio das novas tecnologias, que tem sido capaz de elevar o padrão básico de vida de todos os seres-humanos. Para Klaus Schwab estamos vivendo a 4a Revolução Industrial, diferente de todas as outras, "caracterizada por uma série de novas tecnologias que estão fundindo os mundos físico, digital e biológico, impactando todas as disciplinas, economias e indústrias, e até mesmo desafiando ideias sobre o que significa ser humano".

Palmer e Blake (2018) e Friedman (2017) por sua vez o chamam, respectivamente, de Era da Digitalização, Automação e Era das Acelerações, pois "as três maiores forças do planeta - a tecnologia, a globalização e a mudança climática - estão todas acelerando ao mesmo tempo" (FRIEDMAN, 2017, p. 47), consequentemente, sociedades, ambientes de trabalho e geopolíticas estão em constante mutação e precisam ser repensados.

O Institute for the Future juntamente com o Instituto de Pesquisa da Universidade de Phoenix, em sua pesquisa intitulada Future Work Skills 2020, de autoria de Anna Davies, Devin Fidler e Marina Gorbis listaram seis drivers responsáveis por essas mudanças, com suas respectivas consequências. São eles:

- Longevidade - a expectativa de vida das pessoas está cada vez mais maior, o que por sua vez aumenta o range da população ativa seja trabalhando ou socialmente.

- Máquinas Inteligentes - com o avanço da inteligência artificial, muitas das tarefas antes analógicas agora são feitas por máquina, o que gera ainda mais dados.

- Poder Computacional - os computador e dispositivos eletrônicos tiveram sua capacidade de armazenamento, velocidade e processamento aumentados de forma exponencial.

- Novas Mídias - até alguns anos atrás elas simplesmente não existiam e hoje são responsáveis por gerar uma grande parte do conteúdo da web, vide instagram, tiktok, e até club house lançado mais recentemente.

- Empresas super estruturadas - com o avanço das tecnologias sociais, as organizações são mais conectadas umas com as outras. Atualmente a empresa não opera somente dentro das suas 4 paredes. Ela é capaz de se comunicar e trocar informações através de sistemas globais. 
- Mundo conectado - Atualmente o mundo não se resume a apenas Estados Unidos e Rússia. Países antes menores e sem presença global hoje já são capazes de desenvolver tecnologia, e causar impactos geopolíticos, vide Primavera Árabe, Síria, China, etc.

Devido ao rápido avanço das tecnologias digitais e exponenciais, "nós evoluímos de um mundo de escassez para um de abundância do conhecimento. " (PALMER; BLAKE, 2018) e isso traz consequências diretas para a quantidade de informação nos quais somos expostos: ela está mudando constantemente e mesmo a pessoa mais bem informada já não consegue acompanhar (ARBESMAN, 2012). Arbesman (2012) acrescenta que o conhecimento visto de forma agregada tem uma meia-vida previsível: o tempo necessário para que metade daquele corpo de conhecimento fique obsoleto, ou seja substituído. Parrish (2018) complementa que à medida que nossas ferramentas e conhecimentos se tornam mais avançados, podemos descobrir mais - às vezes coisas novas que contradizem o que pensávamos saber, às vezes nuances sobre coisas antigas. Às vezes, descobrimos uma área inteira que não conhecíamos.

Tal rapidez torna-se um grande desafio para as empresas também. Em muitas delas, a velocidade da mudança no âmbito externo acaba sendo muito maior que internamente, gerando um enorme gap, que pode levar ao fim das mesmas. Singularity University (2018) afirma que isso pode ser percebido no turnover das empresas nas listas S\&P 500 e o Fortune 500. Em média, uma empresa S\&P 500 está sendo substituída a cada 2 semanas e mais de $40 \%$ das empresas que hoje estão na S\&P 500 deixarão de existir dentro de 10 anos.

Em seu relatório 2017 Global Human Capital Trends, Deloitte conclui que para acelerar essa taxa de mudança interna e garantir a sobrevivência das organizações, as mesmas precisam repensar a forma como fazem negócio, como tirar vantagem das tecnologias digitais e também como criar uma cultura de aprendizado contínuo, adaptabilidade, crescimento e desenvolvimento profissional.

Em resumo, precisa-se aumentar sua capacidade de adaptação. O aumento da adaptabilidade humana, por sua vez, de acordo com Astro Teller, CEO da X Development LLC, antiga Google X, consiste em "otimizar o aprendizado", ou seja, aplicar características que canalizem a inovação tecnológica para nossas estruturas culturais e sociais (FRIEDMAN, 2017). Fato é que as pessoas não estão preparadas para o que está por vir. Dados de um estudo da Mckinsey sobre Reskilling e Upskilling da força de trabalho, mostra que para mitigar esse problema, $62 \%$ dos executivos consideram que devem retreinar pelo menos $25 \%$ dos seus colaboradores (PALMER e BLAKE, 2018). Então, como otimizar o aprendizado se nossos modelos de aprendizagem estão defasados?

\subsection{Conectivismo e o Aprendizado em Rede}

Segundo Mitra (2013), o modelo de educação que conhecemos hoje foi útil há 300 anos atrás para construção do império britânico, onde era necessário preparar pessoas em massa para dominar o planeta. Agora o contexto social é outro, com problemas diferentes. Antes, as 
respostas eram encontradas de forma mais fácil. Em em contextos complexos, as respostas corretas não são descobertas facilmente. É necessário investigar, dar sentido e então responder. (SNOWDEN; BOONE, 2007). Para isso, a inteligência coletiva e a diversidade de visões são necessárias, pois, o aprendizado não é construído, ele simplesmente acontece por meio das conexões e redes que formamos (SIEMENS, 2005; MITRA, 2013). E à medida que movemos para essa sociedade cada vez mais em rede, conectada digitalmente, o planejamento e a tomada de decisão central são muito lentos e ineficazes, especialmente para situações complexas (JARCHE, 2017), e isso impacta diretamente como educamos.

Diante do exposto, é necessário repensarmos nossas teorias de aprendizagem. Para Siemens (2005), behaviorismo, cognitivismo e construtivismo, que são três das teorias de aprendizagem mais utilizadas na criação de ambientes instrucionais e que tentam explicar como as pessoas aprendem, foram desenvolvidas em um momento em que a aprendizagem não foi afetada pela tecnologia e "sustentam a noção de que conhecimento é um objetivo (ou um estado) atingível (se não já inato) através de raciocínio ou experiências" (2005, p.2).

Portanto, na tentativa de explicar uma teoria de aprendizagem que melhor correspondesse ao mundo atual, ele criou o Conectivismo, que possui os seguintes princípios (Siemens, 2005, p.5):

1. Aprendizagem e conhecimento apoiam-se na diversidade de opiniões.

2. Aprendizagem é um processo de conectar nós especializados ou fontes de informação.

3. Aprendizagem pode residir em dispositivos não humanos.

4. A capacidade de saber mais é mais crítica do que aquilo que é conhecido atualmente.

5. É necessário cultivar e manter conexões para facilitar a aprendizagem contínua.

6. A habilidade de enxergar conexões entre áreas, ideias e conceitos é uma habilidade fundamental.

Para Siemens (2005), portanto, "criar, preservar e utilizar o fluxo da informação deve ser uma atividade organizacional chave" (p.5), tal como o duto do petróleo o é para o escoamento do mesmo. Cabe à organização mais do que prover treinamentos formais para seus colaboradores, promover uma ecologia de aprendizagem que permita que as conexões aconteçam e o aprendizado flua por meio dos "rios" de saberes. Ou seja, "a aprendizagem está em todo lugar. Aprender e trabalhar estão interconectados na era da rede" (JARCHE, 2017).

Neste cenário, a organização perde o comando/controle da aprendizagem, passa a ser a facilitadora e o indivíduo passa a ser o grande responsável pelo seu próprio desenvolvimento. Num mundo tão dinâmico como o nosso, não é possível mais esperar que organizações acompanhem de forma imediata as mudanças que ocorrem. É necessário tomar o controle do seu aprendizado. Mas como fazer isso, se afinal sempre estamos tão acostumados a receber de forma empurrada o conhecimento, e a depender que as organizações digam para onde tínhamos que ir e o que devemos aprender? 


\subsection{Gestão do Conhecimento}

Seufert et al (1999) e Siemens (2006), afirmam, respectivamente, que o "conhecimento nunca é ocioso" (SEUFERT et al, 1999, p.18), e que a "visão conceitual do conhecimento estático, organizado e definido por 'experts', está sendo substituída por uma visão mais dinâmica e multifacetada" (SIEMENS, 2006, p.3), onde "flui continuamente de atividade para atividade, de pessoa para pessoa, de tarefa para tarefa e de sistema para sistema" (SEUFERT et al, 1999, p.18).

Como forma de entender melhor essas relações, provou-se ser útil criar categorias genéricas dos processos de conhecimento. Em primeiro lugar, é possível dividir a gestão do conhecimento em fases lógicas de evolução através dessas categorias. Em segundo lugar, essas categorias ajudam a identificar barreiras de conhecimento e a desenvolver medidas de aprimoramento do conhecimento. Os processos de conhecimento podem ser classificados em três categorias (Vassiliadis et al., 1999): Localização e Captura; Compartilhamento e Transferência; e Criação.

Von Krogh e Venzin afirmam, no entanto, que a chave para a obtenção da vantagem competitiva de longo prazo não se encontra na gestão do conhecimento existente, mas na capacidade de gerar constantemente novos conhecimentos, e passar para novos produtos e serviços (apud SEUFERT et al, 1999).

Para Nonaka e Takeuchi (2008), o conhecimento dentro de uma organização é criado através da conversão do conhecimento tácito em conhecimento explícito e vice-versa, através de quatro modos de conversão de conhecimento:

1) Socialização: de tácito para tácito;

2) Externalização: de tácito para explícito;

3) Combinação: de explícito para explícito;

4) Internalização: de explícito para tácito.

Ampliando o modelo da espiral, o conhecimento passa do nível individual para o organizacional da seguinte forma (Nonaka e Takeuchi, 2008):

1) Socialização: indivíduo para indivíduo.

2) Externalização: indivíduo para grupo.

3) Combinação: grupo para organização.

4) Internalização: organização para indivíduo.

Confirma-se a importância do conhecimento ao analisar o seu impacto no valor de mercado das organizações. Elsten e Hill (2017), mostram que o papel dos intangíveis tem sido cada vez maior: em 1975, representavam $17 \%$ do valor de mercado das S\&P 500, uma carteira das 500 maiores empresas negociadas nas bolsas americanas NYSE e NASDAQ, e em 2015, 84\%, ou seja, um aumento de quase 5 vezes. "Embora a ênfase maior desses números seja de ativos intangíveis impulsionados pela tecnologia, tais como patentes e segredos comerciais, o valor 
da marca também é um importante componente do estudo". (ELSTEN; HILL, 2017, p.245). O estudo também sugere que o papel dos intangíveis no valor de mercado da organização impacta diretamente no nível de inovação das mesmas, e Gaze (2013), conclui que as empresas mais inovadoras normalmente são mais sucedidas que seus pares.

O conhecimento sendo, portanto, um importante fator de produção, um dos principais responsáveis pelo crescimento econômico (CAVALCANTI; GOMES, 2001), e fonte de vantagem competitiva (von KROGH et al 1999; NONAKA, 2007), tornou-se necessário criar modelos que pudessem ajudar a lidar com os ativos intangíveis. Os pioneiros da Gestão do Conhecimento nesse campo foram Sveiby (1998), Stewart (1998) e Edvinsson (1998), no qual propõem o modelo formado por três componentes básicos (CAVALCANTI; GOMES, 2001), com variações entre eles, conforme mostrado na tabela 1.

\begin{tabular}{|c|c|c|c|c|}
\hline $\begin{array}{c}\text { Componente } \\
\text { Básico }\end{array}$ & Definição comum & $\begin{array}{l}\text { Sveiby } \\
\text { (1998) }\end{array}$ & $\begin{array}{c}\text { Stewart } \\
(1998)\end{array}$ & $\begin{array}{c}\text { Edvinsson } \\
\text { (1998) }\end{array}$ \\
\hline 10 Capital & $\begin{array}{l}\text { Patentes, conceitos, } \\
\text { modelos } \\
\text { administrativos e } \\
\text { informatizados de } \\
\text { uma organização. }\end{array}$ & $\begin{array}{l}\text { Estrutura } \\
\text { Interna }\end{array}$ & $\begin{array}{l}\text { Capital } \\
\text { Estrutural }\end{array}$ & $\begin{array}{c}\text { Capital } \\
\text { Organizacional }\end{array}$ \\
\hline 2ㅇ Capital & $\begin{array}{l}\text { Capacidade } \\
\text { individual de atuação } \\
\text { de cada integrante } \\
\text { da empresa. Ex.: } \\
\text { habilidades, } \\
\text { educação formal, } \\
\text { experiências e } \\
\text { valores de um } \\
\text { determinado } \\
\text { indivíduo. }\end{array}$ & Competências & $\begin{array}{l}\text { Capital } \\
\text { Humano }\end{array}$ & $\begin{array}{l}\text { Capital } \\
\text { Humano }\end{array}$ \\
\hline 3음apital & $\begin{array}{l}\text { Clientes, Parceiros, } \\
\text { Fornecedores e a } \\
\text { imagem que a } \\
\text { empresa tem junto a } \\
\text { eles e ao mercado }\end{array}$ & $\begin{array}{l}\text { Estrutura } \\
\text { externa }\end{array}$ & $\begin{array}{l}\text { Capital de } \\
\text { Clientes }\end{array}$ & $\begin{array}{l}\text { Capital de } \\
\text { Clientes }\end{array}$ \\
\hline
\end{tabular}

Tabela 1: Componentes básicos dos Capitais Fonte: CAVALCANTI; GOMES (2001)

Refletindo nas teorias desenvolvidas pelo Sveiby (1998), Stewart (1998) e Edvinsson (1998) e baseado em experiências práticas desenvolvidas no âmbito do Centro de Referência em Inteligência Empresarial da Universidade Federal do Rio de Janeiro (CRIE/COPPE/UFRJ), 
Cavalcanti e Gomes (2000), desenvolveram seu próprio modelo de gestão para empresas na sociedade do conhecimento, denominado "Capitais do Conhecimento" (CAVALCANTI; GOMES, 2001, p.57). Como contribuição adicional aos modelos apresentados, criou-se o Capital Ambiental. Segundo eles, este Capital reside na importância que as organizações precisam ter da sua visão estratégica, ou seja, onde estão e onde querem chegar, como precisam posicionarse no mercado, qual segmento atuar, e como alinhar-se com os clientes. A definição estratégica, por sua vez, pode ser feita através de técnicas tradicionais de planejamento, enquanto que a análise do posicionamento da organização no mercado, eles recomendam que seja feita através da implantação de um sistema de inteligência competitiva que seja composto por pelo menos quatro fases: "Identificação da informação necessária; Coleta; Análise e principalmente, a transmissão dessa informação aos tomadores de decisão." (CAVALCANTI; GOMES, 2001, p.58)

No entanto, não basta apenas a organização conhecer e monitorar seu ambiente externo. É necessário que exista uma harmonia entre todos os capitais para que ela possa ter de fato uma vantagem competitiva, pois segundo Cavalcanti e Gomes (2000), a priori, "não existe um capital mais importante do que o outro. A importância será baseada no tipo de negócio da organização, grau de maturidade e desenvolvimento, mas o importante é que haja a sinergia mencionada. Uma vez definido os capitais e analisada sua situação, a empresa poderá definir sua estratégia de conhecimento.

Por décadas as organizações tem utilizado gestão do conhecimento como técnica de gestão com relativo sucesso e a prova disso é a inclusão da temática em duas importantes normas relacionadas à gestão: ISO 9001:2015, no qual foi introduzido o requisito 7.1.6 Conhecimento organizacional, no qual inclui a gestão desse conhecimento como um requisito obrigatório (MITTELMANN, 2016), e o PMBOK, no qual teve o processo Gerenciar Conhecimento do Projeto adicionado na 6 edição, mostrando assim o foco cada vez maior na gestão do conhecimento ao longo de todo o projeto (BRAINBOK, 2018).

Os motivos dos insucessos, no entanto, são diversos: eles variam desde a perda de interesse por parte dos executivos até à falta de visibilidade dos benefícios para a toda a organização. Além destes, talvez um dos principais, seja a resistência à mudança cultural que a gestão do conhecimento inevitavelmente traz para a organização como um todo. (MITTELMANN, 2016).

Como solução para alcançar essa cultura favorável ao conhecimento cujo benefício da gestão do conhecimento seja sustentado, Mittelmann (2016) sugere que as organizações devam considerar, como ponto de partida, a introdução da gestão do conhecimento à nível pessoal para todos os seus colaboradores, resultando numa melhoria da performance diária assim como na mudança gradual da cultura de conhecimento na organização como um todo, tornando-se assim um fator crucial na implementação da gestão do conhecimento organizacional, afinal, "o único conhecimento que pode ser gerenciado é o nosso, portanto, a gestão do conhecimento organizacional deve primeiro apoiar o domínio do conhecimento pessoal” (JARCHE, 2017). 


\subsection{Gestão do Conhecimento Pessoal}

Historicamente, o foco da gestão do conhecimento tem sido no conhecimento organizacional e como explorá-lo, tendendo a ignorar o componente pessoal do mesmo, fato este, potencializado pela perspectiva negativa da associação do conhecimento com poder, fazendo com que os indivíduos tenham nenhum incentivo para compartilhar. (WRIGHT, 2005; LEITE; COSTA, 2007; PAULEEN, 2009).

No entanto, isso tende a mudar. Conforme já mostrado anteriormente, os impactos gerados pela era das acelerações exigem uma nova postura tanto dos indivíduos como das empresas, pois "cada vez mais as organizações dependem das contribuições dos trabalhadores do conhecimento" (WRIGHT, 2007, p.2). Sendo assim, "a responsabilidade pelo autodesenvolvimento está agora nas mãos do indivíduo que cada vez mais controla o desenvolvimento da sua carreira e destino. " (PwC, 2018; PAULEEN, 2009, p.222). Richard K. Miller, chama isso de "aprendizagem expedicionária" (FRIEDMAN, 2017).

Mittelmann (2016) ainda acrescenta que os trabalhadores do conhecimento precisam ser capazes de resolver problemas não-padronizados baseados em seus conhecimentos e habilidades utilizando um leque de ferramentas analíticas que são abstratas por natureza, utilizando-se das ferramentas da Web 2.0 para auxilia-los na aquisição, categorização, classificação, armazenamento e compartilhamento do conhecimento deles com suas respectivas comunidades de prática. Isso demandará deles flexibilidade, criatividade e inovação para serem bem-sucedidos nesse ambiente.

Cheong e Tsui (2011) fizeram uma revisão crítica dos modelos de Gestão do Conhecimento Pessoal e baseado na análise deles, concluíram que é claro o quanto a Gestão do Conhecimento Pessoal evoluiu de algo meramente individual e focado na gestão da informação para algo mais orientado a resultados e competências pessoais, dar mais sentido (sensemaking) e autorreflexão.

No entanto, Jarche (2017) pontua que desenvolver a Gestão do Conhecimento Pessoal requer tempo, esforço e reflexão e dentre os principais desafios, destaca a dificuldade cada vez maior de dar sentido às coisas que acontecem ao nosso redor, entendendo as questões complexas envolvidas nisso. Isso deve-se principalmente à nossa dificuldade de separarmos o que é importante do que não tem utilização, pois o que é inútil hoje pode ser útil amanhã. É o desenvolvimento dessas técnicas que requerem tempo de prática e aprendizado. Além disso, para ele, a Gestão do Conhecimento é de pouco valor a menos que os resultados sejam compartilhados conectando-se a outros e contribuindo para conversas significativas. O todo é maior que a soma das partes à medida que construímos sobre o conhecimento dos outros (JARCHE, 2017, p.6)

Portanto, "como trabalhadores do conhecimento ou cidadãos, a PKM é a nossa parte do contrato de aprendizagem social. Sem uma PKM efetiva no nível individual, o aprendizado social tem menos valor. (JARCHE, 2017, p.6)" 


\subsection{Personal Knowledge Mastery}

É necessário reconhecer a importância teórica desses diversos modelos apresentados, fornecendo o fundamento necessário para entender melhor a gestão do conhecimento pessoal. Porém, para efeito prático, "um paradigma menos complexo é necessário para ser facilmente entendido e pronto para ser usado por todos no local de trabalho e a estrutura Seek Sense Share de Jarche procura atender a essas demandas" (MITTELMANN, 2016, p.120). Por meio de "padrões simples facilitados com um toque leve permite que os trabalhadores do conhecimento capturem, interpretem e compartilhem seus conhecimentos (JARCHE, 2017, p.10).

De forma resumida, trata-se de como buscamos as informações e nos mantemos atualizados (Seek); como personalizamos, damos sentido e adicionamos valor à informação (Sense), e por último, como compartilhamos recursos, ideias e experiências com nossa rede (Share).

Como já mencionado anteriormente, para Jarche (2017), "aprendizagem é trabalho e o trabalho é aprendizagem" (p.12), por isso, PKM é a base para a aprendizagem no local de trabalho e na vida pessoal na era das redes. Ela é um "um processo facilitador que ajuda a distribuir poder e conhecimento. A gestão efetiva do conhecimento depende realmente de processos efetivos de PKM" (p.7).

Sendo assim, Jarche (2017) elaborou um modelo que considera efetivo na conexão entre a Gestão do Conhecimento Pessoal e a Gestão do Conhecimento organizacional, divididos entre três níveis independentes, com "PKM para indivíduos, Gestão do Conhecimento para equipes de trabalho e Gestão do Conhecimento Organizacional com foco em curadoria do conhecimento coletivo de indivíduos e equipes" (p.7), conforme mostrado abaixo.

No primeiro nível da Gestão do Conhecimento Pessoal, esta deve ser praticada por todos, concentrando-se no conhecimento implícito, onde o formato seja solto para ser mais pessoal, assim como não seja forçado, cabendo à organização apenas apoiar a prática para que o conhecimento seja compartilhado.

No segundo nível, de gestão do conhecimento para o grupo, o método de narrar o trabalho é mais recomendado, de forma a garantir o maior entendimento possível do grupo. Cabe ao grupo, no entanto, tratar as exceções e aspectos importantes que precisem ser registrados como aprendizado. E é isso que o terceiro nível trata: conhecimento explícito e curadoria do conhecimento.

Jarche (2017), conclui então que, ao adotar essa prática, a organização acaba encorajando a inovação por meio do fluxo do compartilhamento do conhecimento, do trabalho narrado, e por aceitar que as ideias e o entendimento estão em constante construção e que, portanto, não existe um formato perfeito. O mesmo já é feito, por exemplo, há décadas na comunidade dos desenvolvedores e programadores, no qual compartilham em seus fóruns e wikis o trabalho que estão fazendo e os respectivos resultados, ajudando desta forma outros 
profissionais que precisam fazem o mesmo tipo de trabalho, tornando assim a comunidades deles mais inteligente. As empresas precisam fazer o mesmo, pois assim se tornarão mais efetivas ao responder mais rapidamente os problemas que surgirem, criando valor para o negócio (MITTELMANN, 2016).

\section{CONSIDERAÇÕES FINAIS}

No presente artigo, uma questão foi fundamental na investigação: Como a prática da gestão do conhecimento pessoal pode contribuir para o desenvolvimento pessoal, profissional e a resiliência na era digital?

Ao longo do referencial teórico buscou-se responder aos objetivos intermediários para se chegar ao objetivo final, conforme mostrado a seguir:

\subsection{Discorrer sobre o contexto atual que vivemos: a era das acelerações e da abundância e seus desafios.}

Como vimos, o volume e rapidez no qual somos submetidos às informações atualmente chega a ser assustador. Atualmente estamos expostos à conhecimentos e mídias que antigamente não existiam ou estavam sob o controle e acesso de poucos, e isso fazia com que fossemos mais passivos do que ativos na busca do conhecimento, do desenvolvimento pessoal, profissional e até da sociedade.

\subsection{Mostrar como esse contexto atual está impactando na forma como educamos e aprendemos.}

No entanto, esse cenário mudou e o indivíduo tem cada vez mais a responsabilidade e o poder de moldar o seu futuro e o do ambiente que ele vive. Como o Design Thinker André Bello diz: "o ser humano é a Unidade Mínima de Transformação" (BELLO, 2018). Com um post nas redes sociais, ele é capaz de gerar transformações inimagináveis a alguns anos atrás, gerando significativos impactos ao ponto de especialistas compararem a mudança que estamos vivendo como de fato uma mudança de era.

Isso reflete diretamente em nossos sistemas sociais, em como educamos, e em como aprendemos por exemplo. A velocidade da mudança é maior do que podemos acompanhar e a única forma que temos para não cair é continuar pedalando, tal como andar de bicicleta, ou seja, aprender e nos desenvolver continuamente e em rede, pois como vimos o nível de complexidade que enfrentamos atualmente torna proibitivo depender de apenas uma pessoa, ou de um centro tomador de decisão. É necessário explorar o poder das relações sociais na criação de novos conhecimentos, compartilhamento de experiências, e criação de valor. 


\subsection{Contextualizar a gestão do conhecimento e sua importância na aprendizagem.}

Para fazer isso de forma efetiva, seja você uma organização ou indivíduo é necessário mapear e entender seus principais capitais do conhecimento. Nesse contexto, a Gestão do Conhecimento é fundamental, pois é capaz de fornecer a ferramenta intelectual para tanto.

As organizações mais maduras perceberam a importância da gestão do conhecimento organizacional para gerar vantagem competitiva e sustentabilidade dos seus negócios, porém o mesmo não se pode falar dos indivíduos, seja pela falta de conhecimento ou por associarem à práticas organizacionais. Até mesmo as empresas, que na maioria das vezes só o fazem a nível organizacional e não chegam no indivíduo, geralmente por fatores culturais. Por isso, percebese a necessidade de estudar a gestão do conhecimento à nível pessoal.

\subsection{Apresentar o que é a gestão do conhecimento pessoal e seus principais modelos.}

A importância da gestão do conhecimento pessoal, no entanto, já foi tema de diversos autores e renomados especialistas dentro e fora da área de gestão do conhecimento, seja para contribuir para a própria gestão do conhecimento organizacional ou para o próprio desenvolvimento e gestão pessoal e profissional do indivíduo. A forma como ela é feita também varia de autor para autor, e na realidade, é muito individual de cada um. Cada pessoa tem a sua melhor forma de fazer sua Gestão do Conhecimento Pessoal. O que importa é ter em mente o objetivo da mesma.

Para concluir, é importante enfatizar que com a constante mudança que somos expostos, não é possível prever com exatidão o que vai acontecer. Portanto, uma efetiva gestão do conhecimento pessoal é necessária para que possamos nos manter atualizados e atentos ao que está acontecendo ao mesmo tempo que nos dá ferramentas para que possamos rapidamente mudar de direção caso seja necessário.

\section{REFERÊNCIAS}

ARBESMAN, Samuel. The Half-Life of Facts: Why Everything We Know Has an Expiration Date. 1. ed. Editora Penguin Group, 2012. 256 p.

BELLO, Andre. Projeto I - Organizações exponenciais: metodologia CRIE. Rio de Janeiro, CRIE/COPPE/UFRJ, 2018.

BOUD, David. Enhancing Learning Through Self-Assessment. New York: Routledge Falmer, 1995. 
BRAINBOK. Gerenciar o conhecimento do projeto. 2018. Disponível em:

https://www.brainbok.com/guide/pmp/project-integration-management/manage-projectknowledge/. Acesso em 3 mar. 2019.

CAVALCANTI, Marcos; GOMES, Elisabeth. Inteligência Empresarial: Um Novo Modelo de Gestão para a Nova Economia. Produção, v. 10, n. 2, 2001. Disponível em: http://www.scielo.br/pdf/prod/v10n2/v10n2a05 . Acesso em: 25 fev.2019

CHEONG, Ricky K. F.; TSUI, Eric. From Skills and Competencies to Outcome-based Collaborative Work: Tracking a Decade's Development of Personal Knowledge Management (PKM) Models. Knowledge and Process Management, v. 18, n. 3, p. 175-193, 2011. DOI 10.1002/kpm.380. Disponível em: https://onlinelibrary.wiley.com/doi/full/10.1002/kpm.380. Acesso em: 4 mar. 2019.

CHEONG, Ricky K.F.; TSUI, Eric. The roles and values of personal knowledge management: an exploratory study. VINE: The journal of information and knowledge management systems, [S. I.], ano 2010, v. 40, n. 2, p. 204-227, 12 mar. 2010. Disponível em: https://www.emeraldinsight.com/doi/abs/10.1108/03055721011050686. Acesso em: 4 mar. 2019.

DAVIES, Anna; GORBIS, Marina; FIDLER, Devin. Future Skills 2020: The Re-working of "Work". Institute for the Future for the University of Phoenix Research Institute, 2011. Disponível em: http://www.iftf.org/uploads/media/SR-1382A UPRI future work skills sm.pdf. Acesso em: 4 mar. 2019.

DELOITTE UNIVERSITY PRESS. Rewriting the rules for the digital age 2017 Deloitte Global Human Capital Trends. Reino Unido: Deloitte University Press, 6 mar. 2019. Disponível em: https://www2.deloitte.com/cn/en/pages/human-capital/articles/global-human-capitaltrends-2017.html. Acesso em: 6 mar. 2019

DESJARDINS, Jeff. How much data is generated each day?. [S. I.], 15 abr. 2019. Disponível em: https://www.visualcapitalist.com/what-happens-in-an-internet-minute-in-2019/. Acesso em: 12 maio 2019.

What Happens in an Internet Minute in 2019?. [S. I.], 13 mar. 2019. Disponível em: https://www.visualcapitalist.com/what-happens-in-an-internet-minute-in-2019/. Acesso em: 12 maio 2019.

DIAMANDIS, Peter H.; KOTLER, Steven. Abundância: O Futuro é melhor do que imagina. 1. ed. São Paulo: HSM Editora, 2012.

ELSTEN, Cate; HILL, Nick. Intangible Asset Market Value Study?. Les Nouvelles - Journal of the Licensing Executives Society, v. LII, n. 4, 2017. Disponível em:

https://papers.ssrn.com/sol3/papers.cfm?abstract id=3009783. Acesso em: 3 mar. 2019.

FORTE, Tiago. Building a Second Brain: An Overview. [S. I.], 20 fev. 2019. Disponível em: https://praxis.fortelabs.co/basboverview/. Acesso em: 13 maio 2019. 
FRIEDMAN, Thomas L.Obrigado pelo atraso: Um guia otimista para sobreviver em um mundo cada vez mais veloz. 1. ed. Editora Objetiva, 2017.

GAZE, Laura. Innovative organizations with intangible assets are more successful than counterparts. Reuters, 8 out. 2013. Disponível em: https://www.reuters.com/article/usinnovative-organizations-idUSBRE99707E20131008. Acesso em: 5 mar. 2019

HERBERT, Brian et al. Dune: House Harkonnen. [S. I.]: Listening Library, 2000.

JARCHE, Harold. Finding perpetual beta: Reflections On The Network Era. 2014.

Sense-making with PKM. [S. I.: s. n.], 12 mar. 2009. Disponível em:

http://jarche.com/2009/03/sense-making-with-pkm/. Acesso em: 4 mar. 2019.

The PKM Handbook Personal Knowledge Mastery. 2017.

Work is learning and learning is the work - Interview with Harold Jarche -

Canadian Consultant and Keynote Speaker at this year's HR Innovation Day. Leipziger-HRMBlog, [S. I.], 30 abr. 2019. Disponível em: http://leipzig-hrm-

blog.blogspot.com/2019/04/work-is-learning-and-learning-is-work.html. Acesso em: 12 maio 2019.

KINSINGER, Paul; WALCH, Karen. Living and Leading in a VUCA World. Disponível em: http://www.forevueinternational.com/Content/sites/forevue/pages/1482/4_1_Living_and _Leading_in_a_VUCA_World_Thunderbird_School.PDF. Acesso em: 26 fev. 2019

LAMBE, Patrick. Personal Knowledge Management: a DIY Guide to Knowledge Management - Part 2. 2002. Disponível em: http://www.greenchameleon.com/thoughtpieces/pkm.pdf. Acesso em: 4 mar. 2019.

LEITE, F. C. L.; COSTA, S. M. S. Gestão do conhecimento científico: proposta de um modelo conceitual com base em processos de comunicação científica. Ci. Inf., Brasília, v. 36, n. 1. 2007. Disponível em: http://www.scielo.br/pdf/ci/v36n1/a07v36n1.pdf. Acesso em:

LÉVY, Pierre. Do Hipetertexto opaco ao hipertexto transparente - Parte 1. In: SIMPÓSIO HIPERTEXTO E TECNOLOGIAS NA EDUCAÇÃO: REDE SOCIAIS E APRENDIZAGEM, 3., 2010, Recife, PE. Anais [...]. 2011. Disponível em:

https://www.youtube.com/watch?v=ZLwgyuiORxw\&list=PL6BC4E401E5307E4C\&index=1. Acesso em: 12 maio 2019.

LODI, J. B. Resenha: - The age of discontinuity - Guidelines to out changing society - (Peter F. Drucker -1969). Revista de Administração de Empresas, v. 9, n. 3, 1969

MADEIRA, Gilberto dos Santos; PEREIRA, Moacir. Conhecimento e Decisão: A Gestão do Conhecimento Pessoal para a Tomada de Decisões Corporativas. Revista Gestão Industrial, Ponta Grossa, PR, v. 6, n. 4, p. 38-69, 20 maio 2019. DOI 10.3895/S1808-

04482010000400003. Disponível em:

https://periodicos.utfpr.edu.br/revistagi/article/view/672. Acesso em: 12 mai. 2019. 
MARTINS, Amilton Rodrigo de Quadros et al.. Uso de Design Thinking como Experiência de Prototipação de Ideias no Ensino Superior. Future Studies Research Journal: Trends and Strategies, v. 8, n. 1, p. 208-224, 2016. Disponível em:

http://www.spell.org.br/documentos/ver/41982/uso-de-design-thinking-como-experienciade-prototipacao-de-ideias-no-ensino-superior/i/pt-br. Acesso em: 22 mai. 2019.

MITRA, Sugata. BUILD a School in the Cloud. TED Conferences, 27 de fevereiro de 2013. Disponível em: https://www.ted.com/talks/sugata_mitra_build_a_school_in_the_cloud. Acesso em: 4 mar. 2019.

MITTELMANN, Angelika. Personal Knowledge Management as Basis for Successful Organizational Knowledge Management in the Digital Age. Procedia Computer Science, v. 99, p. 117 - 124, 2016. DOI https://doi.org/10.1016/j.procs.2016.09.105. Disponível em: https://www.sciencedirect.com/science/article/pii/S1877050916322505. Acesso em: 4 mar. 2019

NONAKA, Ikujiro. The Knowledge-Creating Company. Harvard Business Review, 2007. Disponível em: https://hbr.org/2007/07/the-knowledge-creating-company Acesso em: 04 mar. 2019

NONAKA, Ikujiro; TAKEUCHI, Hirotaka. Gestão do Conhecimento. Tradução: Ana Thorell. 1. ed. Porto Alegre: Editora Bookman, 2008.

OSTERWALDER, A.; PIGNEUR, Y. Business Model Generation - Inovação em Modelos de Negócios: um manual para visionários, inovadores e revolucionários. Rio de Janeiro, RJ: Atlas Books, 2011.

PALMER, Kelly; BLAKE, David.The Expertise Economy: How the smartest companies use learning to engage, compete, and succeed. 1. ed. Editora Nicholas Brealey, 2018.

PARRISH, Shane. Half Life: The Decay of Knowledge and What to Do About It. 1. Farnam Street Media Inc, 2018. Disponível em: https://fs.blog/2018/03/half-life/. Acesso em: 4 mar. 2019

PAULEEN, D. J. Personal knowledge management: putting the "person" back into the knowledge equation. Online Information Review. v. 33, n.02, 2009. Disponível em: https://www.emeraldinsight.com/doi/abs/10.1108/14684520910951177. Acesso em: 02 fev. 2019.

THURLER, Larriza; PIRES, Camila. Kmap Canvas: uma Proposta de Prática para Mapear Conhecimento Aplicando Design Thinking. In: II SIMPÓSIO INTERNACIONAL NETWORK SCIENCE, 2018, Rio de Janeiro. Anais [...]. [S. I.: s. n.], 2018. Disponível em:

http://networkscience.com.br/wpcontent/uploads/2018/11/IISINS_GC_Artigo_KMAPCanvas.pdf. Acesso em: 15 jan. 2019.

PWC. Workforce of the future The competing forces shaping 2030. 2018. Disponivel em: https://www.pwc.com/gx/en/services/people-organisation/workforce-of-the- 
future/workforce-of-the-future-the-competing-forces-shaping-2030-pwc.pdf. Acesso em: 4 mar. 2019.

ROWLEY, Jennifer. The wisdom hierarchy: representations of the DIKW hierarchy. Journal of Information Science, v. 33, n. 2, p. 163-180, 2007. DOI 10.1177/0165551506070706. Disponível em: https://journals.sagepub.com/doi/abs/10.1177/0165551506070706. Acesso em: 4 mar. 2019.

SEUFERT, Andreas ; VON KROGH, Georg; BACH, Andrea. Towards knowledge networking. Journal of Knowledge Management, v. 3, n. 3, 1999. Disponível em: https://www.emeraldinsight.com/doi/abs/10.1108/13673279910288608 . Acesso em: 4 mar. 2019.

SIEMENS, G. Learning and Knowing in Networks: Changing roles for Educators and Designers. 2008. ITFORUM. disponível em

https://www.calvin.edu/ dsc8/documents/GeorgeSiemensPresentation-Jan2008.pdf Acesso em: 01 nov. 2018.

Connectivism: A Learning Theory for the Digital Age. International Journal of Instructional Technology and Distance Learning, v. 2, n. 1, p. 3-10, 01 2005. Disponível em: http://www.itdl.org/Journal/Jan_05/Jan_05.pdf. Acesso em: 4 mar. 2019.

SINGULARITY UNIVERSITY. The Exponential Leader's Guide to Disruption: Disrupt yourself before someone else does. 2018. Disponível em:

https://su.org/resources/ebook/exponential-leaders-guide-to-disruption/. Acesso em 05 mar. 2019.

SNOWDEN, David J.; BOONE, Mary E. A Leader's Framework for Decision Making. Harvard Business Review, 11 2007. Disponível em: https://hbr.org/2007/11/a-leaders-framework-fordecision-making. Acesso em: 4 mar. 2019.

SOUSA, Priscila Aparecida; RAMOS FILHO, Américo da Costa; VASCONCELOS, Carlos Henrique de. Gestão do Conhecimento Pessoal: Definições e Proposições para uma Nova Agenda de Pesquisa. In: XI SIMPÓSIO DE EXCELÊNCIA EM GESTÃO E TECNOLOGIA, 2014, Rio de Janeiro, RJ. Anais [...]. Rio de Janeiro: [s. n.], 2014. Disponível em: https://www.aedb.br/seget/arquivos/artigos14/42220477.pdf. Acesso em: 18 dez. 2018.

SOUTO, Leonardo Fernandes. Coaching E Gestão Do Conhecimento Pessoal. Rio de Janeiro, RJ: Synergia, 2017.

ŚWIGOŃ, Marzena. Personal knowledge and information management - conception and exemplification. Journal of Information Science, [S. I.], 19 set. 2013. Disponível em: https://journals.sagepub.com/doi/abs/10.1177/0165551513501435?journalCode=jisb. Acesso em: 13 mai.2019.

Personal Knowledge Management - a set of skills and methods. History - Archive studies - Information Science: methodological issues, [S. I.], p. 207-213, 2010. Disponível em: 
https://www.researchgate.net/publication/268207210_Personal_knowledge_management_ -_a_set_of_skills_and_methods. Acesso em: 12 mai. 2019.

VASSILIADIS, Stefanos et al. Knowledge Networks: Linking Knowledge Management to Business Strategy. Bericht BE HSG , St. Gallen, ano 1999, n. 11, 13 dez. 1999. Disponível em: https://www.alexandria.unisg.ch/214149/1/11_bim.pdf. Acesso em: 4 mar. 2019.

VERGARA, Sylvia Constant. Projetos e Relatórios de Pesquisa em Administração. 2. ed. São Paulo: Atlas,1998.

WORLD ECONOMIC FORUM. The Fourth Industrial Revolution, by Klaus Schwab. [S. I.]. Disponível em: https://www.weforum.org/about/the-fourth-industrial-revolution-by-klausschwab. Acesso em: 4 mai. 2019.

Wright, Kirby. Personal Knowledge Management Planning Guide: developing ways to work smarter not harder. 2007 Disponível em: http://www.knowledgeresources.ca/pkm-planning. Acesso em: 4 mar. 2019.

Personal knowledge management: supporting individual knowledge worker performance. Knowledge Management Research \& Practice, v. 3, 2005. Disponível em: https://www.tandfonline.com/doi/full/10.1057/palgrave.kmrp.8500061. Acesso em: 2 fev. 2019 